\title{
Coherent and incoherent Upsilon production in ultraperipheral collisions at the Large Hadron Collider
}

\author{
M. B. Gay Ducati, F. Kopp, and M. V. T. Machado \\ High Energy Physics Phenomenology Group, GFPAE IF-UFRGS, Caixa Postal 15051, \\ CEP 91501-970, Porto Alegre, Rio Grande do Sul, Brazil
}

(Received 12 July 2017; published 5 September 2017)

\begin{abstract}
The exclusive photoproduction of $\Upsilon(n S)$ states was calculated in ultraperipheral collisions for coherent and incoherent process in $\mathrm{PbPb}$ at $\sqrt{s_{N N}}=5.5 \mathrm{TeV}$. Different dipole models were compared in the theoretical framework of light-cone color dipole formalism. Moreover, we calculated the differential cross section for the Upsilon states and their total cross section for two intervals of rapidity: $|y| \leq 44$ and $2 \leq \mathrm{y} \leq 4.5$. A systematic study is done on the theoretical uncertainties associated to the production, and predictions are presented for the first time for the incoherent cross section of the radially excited states.
\end{abstract}

DOI: 10.1103/PhysRevD.96.054001

\section{INTRODUCTION}

The study of exclusive meson photoproduction in ultraperipheral collisions (UPC) [1] is an essential tool to understand the low-x physics and also to investigate the gluon density in this regime. In the UPC case, the exclusive photoproduction dominates the process through the emission of quasivirtual photons which interact with the target. The photon-target interaction amplitude, when considering the light-cone dipole formalism [2], can be written as a convolution between the photon-meson wave functions' overlap and the elementary dipole-target cross section [3]. In addition, the process considered here is quasielastic, $Q^{2} \approx 0$, and in the region of small-x, the gluon density may increase to the point where gluon fusion, $g g \rightarrow \mathrm{g}$, becomes significant. This kind of fusion produces nonlinear effects in the evolution equations. For instance, at midrapidity the typical value of the Bjorken variable is $x=\frac{m_{\Upsilon}}{\sqrt{s_{A A}}} \simeq 10^{-3}$ for $\mathrm{PbPb}$ collisions at the LHC. Differently from DGLAP equations, which are linear equations, dipole models incorporate linear and nonlinear effects [4]. In pQCD the exclusive meson photoproduction has a differential cross section $\propto$ $\left[x g\left(x, Q^{2}\right)\right]^{2}$, where the gluon distribution functions do not take into account the effects of saturation since they are evolved by DGLAP equations. The inclusion of parton saturation and nuclear effects is crucial in describing even the experimental observations in heavy-ion collisions at the RHIC (for instance, see Ref. [5]). Within the color dipole approach one can introduce information on dynamics beyond the leading logarithmic QCD approach for meson production and computing predictions for the radially excited states is a reasonably easy task [3]. From the experimental point of view, the considered process is quite clear due to the presence of two rapidity gaps. The rapidity gap describes a region between the axis beam and the decay of a meson $\left(e^{+}+e^{-}, \mu^{-}+\mu^{+}\right)$free of particles. In this way, the experimental observation of this kind of quasidiffractive process is facilitated. In addition, the absorption corrections in this case are not strong as in the corresponding final state in proton-proton collisions.

In this work, we investigate the exclusive (coherent and incoherent) production of $\Upsilon(1 S)$ and its radially excited states $\Upsilon(2 S)$ and $\Upsilon(3 S)$ in $\mathrm{PbPb}$ collisions for LHC energy. In a previous work [6], the coherent photoproduction of $\Upsilon$ states at various energies in $p p, \mathrm{pPb}$, and $\mathrm{PbPb}$ collisions at the LHC has been considered. Those calculations were carried out in the theoretical framework of the color light-cone dipole formalism [2] and focused only on the coherent channel where the initial state particles remain intact after interaction. It was shown that the corresponding predictions describe correctly the experimental results from the LHCb Collaboration [7] for $\Upsilon$ photoproduction in $p p$ collisions. Those data were obtained for typically large rapidities and the $x$-values to be covered are increasingly smaller for forward rapidities. We roughly get $x=$ $\frac{m_{\Upsilon}}{\sqrt{S_{A A}}} e^{-y} \simeq 8 \times 10^{-5}$ at $y=3$ and it is clear that gluon dynamics is being probed at extremely low- $x$ and low perturbative scales, $\mu^{2} \simeq 20 \mathrm{GeV}^{2}$. This kinematical range is in the limit of application of usual pQCD and the saturation approach should be relevant. For nuclear targets, the nuclear saturation scale $Q_{\text {sat }, A}^{2} \simeq c A^{1 / 3} Q_{\text {sat }, p}^{2}$ (with $c \simeq 0.3$ ) reaches $2 \mathrm{GeV}^{2}$ in those cases [8]. The main novelty in the current work is the detailed study of the incoherent cross section for the Upsilon states. This is quite important, as it was pointed out that incoherent diffraction probes the fluctuations in the interactions strengths of multiparton Fock states in the nuclear wave functions [9]. The connection between incoherent diffraction and fluctuations is quite a rich subject, and the pioneering works are found in Refs. [10]. Recently the topic has been very active and we call attention to the following works $[11,12]$.

The paper is organized as follows. In the next section we give the main theoretical information to obtain the rapidity distribution of coherent and incoherent production of $\Upsilon(1 S, 2 S, 3 S)$ states in $\mathrm{PbPb}$ collisions for the future 
LHC run and energies close to run 2. The main motivation is the successful description of experimental results measured by the LHCb Collaboration [7] for $\Upsilon(1 S)$ in $p p$ collisions. In Sec. III we present the phenomenological calculations, discuss the main theoretical uncertainties, and perform a comparison with other approaches. For instance, we compare the present calculation to the predictions available using STARlight Monte Carlo [13,14]. Finally, we show the main conclusions.

\section{THEORETICAL FRAMEWORK}

The exclusive meson photoproduction in nucleusnucleus collisions can be factorized in terms of the equivalent flux of photons of the nucleus projectile and photon-target production cross section [1]. In UPCs there is the absence of strong interactions between the projectile particle and the target. In this case the reaction is characterized by impact parameter $>2 R_{A}$ and as the interaction is ultrarelativistic and purely electromagnetic, one can use the Weizsäcker-Williams approximation [1]. The photon energy spectrum, $d N_{\gamma}^{A} / d \omega$, which depends on the photon energy $\omega$, is well known [1]. The rapidity distribution for $\Upsilon$ state photoproduction in $A A$ collisions can be written as

$$
\begin{aligned}
& \frac{d \sigma}{d y}(A A \rightarrow A \otimes \Upsilon(n S) \otimes Y) \\
& \quad=\left[\omega \frac{d N_{\gamma}^{A}}{d \omega} \sigma(\gamma A \rightarrow \Upsilon(n S) Y)+(y \rightarrow-y)\right],
\end{aligned}
$$

where the photon flux in the nucleus is denoted by $d N_{\gamma}^{A} / d \omega$ and $Y=A$ (coherent case) or $Y=A^{*}$ (incoherent case). The symbol $\otimes$ denotes the large rapidity gap between the produced meson and the final state's nucleus.

The produced state with mass $m_{V}$ has rapidity $y \simeq$ $\ln \left(2 \omega / m_{V}\right)$ and the square of the $\gamma A$ center-of-mass energy is given by $W_{\gamma A}^{2} \simeq 2 \omega \sqrt{s}$. The photon-Pomeron interaction will be described within the light-cone dipole frame, where the probing projectile fluctuates into a quark-antiquark pair with transverse separation $r$ (and momentum fraction $z$ ) long after the interaction, which then scatters off the hadron. The cross section for exclusive photoproduction of $\Upsilon$ states off a nucleon target is given by

$\sigma(\gamma p \rightarrow \Upsilon p)=\frac{\left|\sum_{h, \bar{h}} \int d z d^{2} r \Psi_{h, \bar{h}}^{\gamma} \sigma_{\mathrm{dip}}(x, r) \Psi_{h, \bar{h}}^{V *}\right|^{2}}{16 \pi B_{\Upsilon}}$,

where $\Psi^{\gamma}$ and $\Psi^{V}$ are the light-cone wave function of the photon and of the vector meson $(V=\Upsilon)$, respectively. The dipole-proton cross section is denoted by $\sigma_{\text {dip }}(x, r)$ and the diffractive slope parameter by $B_{V}$. In this context, we are implicitly assuming that the proton shape is Gaussian and that the impact parameter dependence factorizes out from the dipole-nucleon scattering amplitude. Here, we consider the energy dependence of the slope using the Regge motivated expression [6].

The exclusive photoproduction off nuclei for coherent and incoherent processes can be simply computed at high energies where the large coherence length $l_{c} \gg R_{A}$ is fairly valid. The expressions for both cases are given by [15]

$$
\begin{aligned}
\sigma(\gamma A \rightarrow \Upsilon A)= & \int d^{2} b \mid \sum_{h, \bar{h}} \int d z d^{2} r \Psi_{h, \bar{h}}^{\gamma} \Psi_{h, \bar{h}}^{V *} \\
& \times\left.\left[1-\exp \left(-\frac{1}{2} \sigma_{\mathrm{dip}}(x, r) T_{A}(b)\right)\right]\right|^{2}, \\
\sigma\left(\gamma A \rightarrow \Upsilon A^{*}\right)= & \int d^{2} b \frac{T_{A}(b)}{16 \pi B_{V}} \mid \sum_{h, \bar{h}} \int d z d^{2} r \Psi_{h, \bar{h}}^{\gamma} \Psi_{h, \bar{h}}^{V *} \\
& \times\left.\sigma_{\mathrm{dip}}(x, r) \exp \left[-\frac{1}{2} \sigma_{\mathrm{dip}}(x, r) T_{A}(b)\right]\right|^{2},
\end{aligned}
$$

where $T_{A}(b)=\int d z \rho_{A}(b, z)$ is the nuclear thickness function. In the numerical evaluations, we have considered the boosted Gaussian wave function and several phenomenological saturation models, which encode the main properties of the saturation approaches. Accordingly, the cross sections above include both the skewedness and real part of amplitude corrections. Namely, we multiply the result above by $K^{2}=R_{g}^{2}\left(1+\beta^{2}\right)$, where $\beta=\tan \left(\pi \lambda_{e f} / 2\right)$ is the ratio of real to imaginary parts of the scattering amplitude and $R_{g}$ incorporates the off-forward correction (see [6] for details). The effective power on energy, $\lambda_{e f}$, is determined for each case. In order to take into account the threshold correction for the dipole cross section, we have multiplied them by a factor $(1-x)^{7}$.

Finally, we set the parameters and phenomenological models to be considered in next section. For the slope parameter we considered the energy dependency from the Regge phenomenology [6],

$$
B_{\Upsilon}=b_{e l}^{\Upsilon}+2 \alpha^{\prime} \log \left(\frac{W_{\gamma A}^{2}}{W_{0}^{2}}\right),
$$

with $\alpha^{\prime}=0.164 \mathrm{GeV}^{-2}, W_{0}=95 \mathrm{GeV}, b_{e l}^{\Upsilon_{(1 S)}}=3.68 \mathrm{GeV}^{-2}$, $b_{e l}^{\Upsilon_{(2 S)}}=3.61 \mathrm{GeV}^{-2}$, and $b_{e l}^{\Upsilon_{(3 S)}}=3.57 \mathrm{GeV}^{-2}$. It will be taken into account only for the incoherent cross section. For the meson wave funtion, we will use the boostedGaussian model [16] because it can be applied in a systematic way for excited states. The corresponding function is given by [17]

$$
\phi_{n S}(r, z)=\left[\sum_{k=0}^{n-1} \alpha_{n S, k} R_{n S}^{2} \hat{D}^{2 k}(r, z)\right] G_{n S}(r, z)
$$

with $\alpha_{n S, 0}=1$. The operator $\hat{D}^{2}(r, z)$ is defined by 

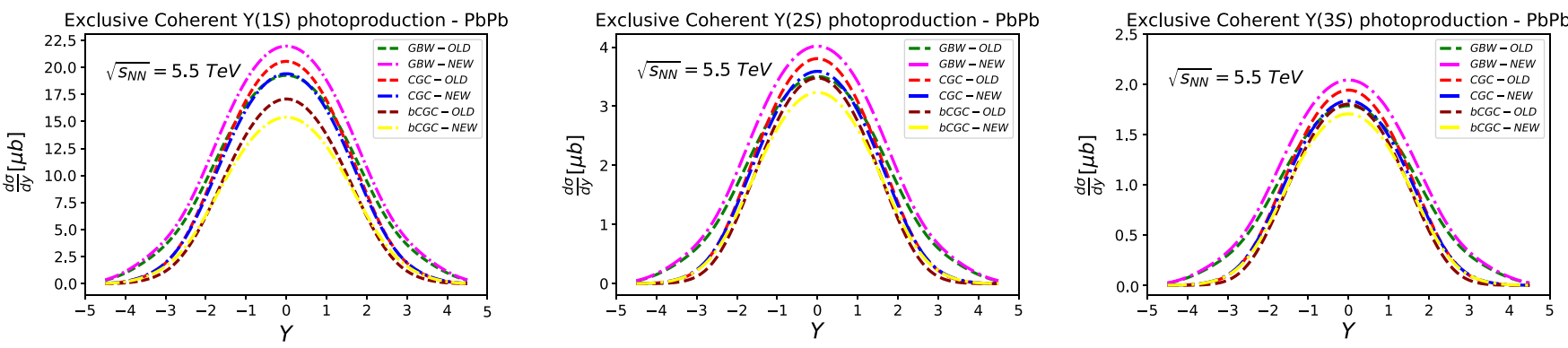

FIG. 1. Exclusive coherent photoproduction of $\Upsilon(1 S, 2 S, 3 S)$ in PbPb at $\sqrt{s}=5.5 \mathrm{TeV}$ for the GBW, CGC, and bCGC dipole models.

$$
\hat{D}^{2}(r, z)=\frac{m_{f}^{2}-\left(\frac{1}{r} \partial_{r}+\partial_{r}^{2}\right)}{4 z(1-z)}-m_{f}^{2}
$$

and it acts on the following generatrix function:

$$
\begin{aligned}
G_{n S}(r, z)= & \mathcal{N}_{n S} z(1-z) \\
& \times \exp \left(-\frac{m_{f}^{2} \mathcal{R}_{n S}^{2}}{8 z(1-z)}-\frac{2 z(1-z) r^{2}}{\mathcal{R}_{n S}^{2}}+\frac{m_{f}^{2} \mathcal{R}_{n S}^{2}}{2}\right) .
\end{aligned}
$$

The main physical quantity is the dipole scattering cross section. We consider the following phenomenological models in our analysis: GBW [18], CGC [19], and BCGC [20]. The GBW model is defined by the eikonal shape for the dipole cross section,

$$
\sigma_{q \bar{q}}^{\mathrm{GBW}}(x, r)=\sigma_{0}\left(1-e^{-r^{2} Q_{s}^{2}(x) / 4}\right),
$$

where $\sigma_{0}=2 \pi R^{2}$ is a constant and $Q_{s}^{2}(x)=\left(x_{0} / x\right)^{\lambda} \mathrm{GeV}^{2}$ denotes the saturation scale. We also consider the CGC model [19], based on the color glass condensate framework, in which gluon saturation effects are incorporated via an approximate solution of the Balitsky-Kovchegov equation [4]. The expression for the CGC model is given by

$$
\sigma_{q \bar{q}}^{C G C}(x, r)=\sigma_{0}\left\{\begin{array}{ll}
\mathcal{N}_{0}\left(\frac{r Q_{s}}{2}\right)^{\gamma_{\text {eff }}(x, r)} & : r Q_{s} \leq 2 \\
1-e^{-A l n^{2}\left(B r Q_{s}\right)} & : r Q_{s}>2
\end{array},\right.
$$

where $\gamma_{\text {eff }}(x, r)=2\left(\gamma_{s}+(1 / \kappa \lambda \ln (1 / x)) \ln \left(2 / r Q_{s}\right)\right)$ is the effective anomalous dimension and one has the constant $\kappa=9.9$.

In order to investigate the theoretical uncertainty associated to the models for the dipole cross section, we use the original values ("OLD" label) of parameters for the fits including the charm contribution. That is, for GBWOLD we follow Ref. [18], for CGC-OLD Ref. [21] is considered, and bCGC-OLD refers to Ref. [20]. The bCGC model uses the same functional form of Eq. (8) and replaces the saturation scale in the following way: $Q_{s}^{2}(x) \rightarrow Q_{s}(x, b)^{2}=\left(x_{0} / x\right)^{\lambda} \exp \left[-b^{2} /\left(2 \gamma_{s} B_{C G C}\right)\right]$. Moreover, we consider the updated version of those models, GBW-NEW [12], CGC-NEW [22], and bCGCNEW [22], respectively. A comment is in order here: the GBW-NEW parametrization is very different from other color dipole fits, as it includes the energy evolution of the subnucleonic shape of the proton and it can potentially significantly affect the incoherent cross section. In particular, GBW-OLD and GBW-NEW are qualitatively very different and GBW-NEW was not fitted to all $F_{2}$ small$x$ data (DESY-HERA) as discussed in detail in Ref. [12].

\section{RESULTS AND DISCUSSIONS}

Let us start the analysis by computing the theoretical predictions for the coherent process for $\mathrm{PbPb}$ collisions at 5.5 TeV. Here, we disregard any absorptive corrections. Figure 1 shows the results for photoproduction of $\Upsilon$ states, including its radial excitations, taking into account the different models presented in the last section. The theoretical uncertainty is relatively large, being of order $15 \%$ for the $1 S$ state (similar for the remaining $2 S$ and $3 S$ states). We could have an additional uncertainty related to the vector meson wave function; however, in Ref. [23] it was shown that this is not the case for $\Upsilon$ states (the overall theoretical uncertainty is within the experimental error bars in the $p p$ case [7]). For the main contribution, we have $d \sigma_{\mathrm{coh}} / d y(y=0)=18.5 \pm 3.5 \mu \mathrm{b}$ for $\Upsilon(1 S)$. The relative contribution of the excited states compared to the bound states is $\Upsilon(1 S) / \Upsilon(2 S) / \Upsilon(3 S)=1 / 0.17 / 0.09$. We see that the relative normalization and the overall behavior is

TABLE I. Integrated cross section (in units of $\mu \mathrm{b}$ ) for coherent reactions, $P b P b \rightarrow P b \Upsilon P b$, for full rapidity coverage (and forward rapidities). Here, we consider the updated versions of dipole cross sections.

\begin{tabular}{lccc}
\hline \hline Process: $\mathrm{PbPb}$ & $\sqrt{s}=5.5 \mathrm{TeV}$ & $|y| \leq 4(2 \leq y \leq 4.5)$ & \\
\hline$\Upsilon(n S)$ & GBW & CGC & b-CGC \\
$\Upsilon(1 S)$ & $163.7(60.8)$ & $171.9(63.8)$ & $143(53.1)$ \\
$\Upsilon(2 S)$ & $20.3(7.8)$ & $22.0(8.2)$ & $20.5(7.7)$ \\
$\Upsilon(3 S)$ & $10.3(3.9)$ & $11.9(4.3)$ & $10.9(4.1)$ \\
\hline \hline
\end{tabular}


TABLE II. Integrated cross section (in units of $\mu \mathrm{b}$ ) for incoherent reactions, $\mathrm{PbPb} \rightarrow P b \Upsilon P b^{*}$, for full rapidity coverage (and forward rapidities). Here, we consider the updated versions of dipole cross sections.

\begin{tabular}{lccc}
\hline \hline Process: $\mathrm{PbPb}$ & $\sqrt{s}=5.5 \mathrm{TeV}$ & $|y| \leq 4(2 \leq y \leq 4.5)$ & \\
\hline$\Upsilon(n S)$ & $\mathrm{GBW}$ & $\mathrm{CGC}$ & $\mathrm{b}-\mathrm{CGC}$ \\
$\Upsilon(1 S)$ & $61.2(25.6)$ & $58.5(24.4)$ & $44.5(18.6)$ \\
$\Upsilon(2 S)$ & $8.2(3.4)$ & $8.0(3.4)$ & $6.9(2.9)$ \\
$\Upsilon(3 S)$ & $4.2(1.8)$ & $4.2(1.8)$ & $3.8(1.6)$ \\
\hline \hline
\end{tabular}

changed mostly at midrapidity when comparing the old and updated versions of the dipole cross sections (the deviation at large rapidities is less evident). Notice that the LHCb data for Upsilon production in $p p$ collisions is reproduced by all the models in the forward region [7] as shown in Ref. [6]. Therefore, the current level of the experimental uncertainties does not allow us to make definitive statements about the precision of the models considered. For the sake of completeness, we present the integrated cross sections considering distinct cuts on rapidity. In Table I, we present the results for the full rapidity coverage, $-4<y<4$, and forward rapidities, $2 \leq y \leq 4.5$. In both Tables I and II, we present only the updated versions of the

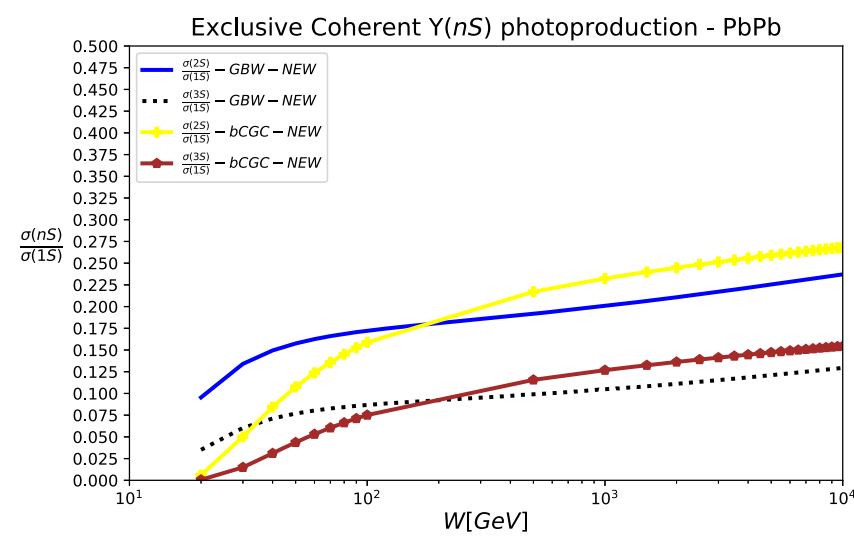

FIG. 2. The ratio of the cross sections, $\sigma(\gamma A \rightarrow \Upsilon(n S) A) /$ $\sigma(\gamma A \rightarrow \Upsilon(1 S) A)$, as a function of $W_{\gamma A}$ for the parametrizations bCGC-NEW and GBW-NEW. dipole cross sections. For the sake of completeness, we present the ratio of the cross sections, $\sigma(\gamma A \rightarrow \Upsilon(n S) A) /$ $\sigma(\gamma A \rightarrow \Upsilon(1 S) A)$, as a function of the photon-nucleus center-of-mass energy, $W_{\gamma A}$. We present in Fig. 2 the result using the bCGC-NEW and GBW-NEW dipole cross sections. It was verified that the CGC-NEW result is quite similar to the bCGC-NEW one. We see a relative energy dependence, following the same trend as for the $\psi(n S)$ states [24].

We now focus on the incoherent reaction, $\mathrm{PbPb} \rightarrow$ $P b \Upsilon P b^{*}$. This is a new contribution to the literature concerning the Upsilon production. The rapidity distribution is shown in Fig. 3 using the same notation as the previous figure. As already known, the incoherent cross section is smaller than the coherent one. The typical ratio is $\left(d \sigma_{\text {inc }} / d y\right) /\left(d \sigma_{\text {coh }} / d y\right) \simeq 0.2$. For instance, we obtain $d \sigma_{\text {inc }} / d y(y=0)=3.75 \pm 1.25 \mu \mathrm{b}$ for $\Upsilon(1 S)$. The theoretical uncertainty seems to be larger than in the coherent case. The integrated cross sections are shown in Table II in the rapidity ranges $|y| \leq 4$ and $2 \leq y \leq 4.5$.

The calculations performed above can be compared to other theoretical approaches available in the literature. Let us start by comparing them to STARlight Monte Carlo [13]. For the coherent production, the predictions for the $\Upsilon$ state ratios are lower than the STARlight results. As discussed in Ref. [6], the possible origin comes from the extrapolation of HERA-DATA and taking a fixed ratio for the distinct states in Monte Carlo, whereas in the current case the evolution on energy is dynamically generated by the parton saturation approach models and mostly by the meson wave functions for the radially excited states. The nuclear effects are also computed in a different way in the two formalisms. In STARlight, the nuclear shadowing is calculated using vector meson dominance (VDM) plus the Glauber model for hadronic collisions. In our case, shadowing comes from the multiple scatterings of color dipoles and is described by the Glauber-Gribov approach. We verified that our results are also smaller that those in Ref. [13], which can be related to more shadowing in color dipole models compared to the $\mathrm{VDM}+$ Glauber approach. In Ref. [25] only the coherent contribution was computed and the theoretical uncertainty we have found in the color dipole approach is comparable to perturbative QCD formalism. Concerning similar dipole
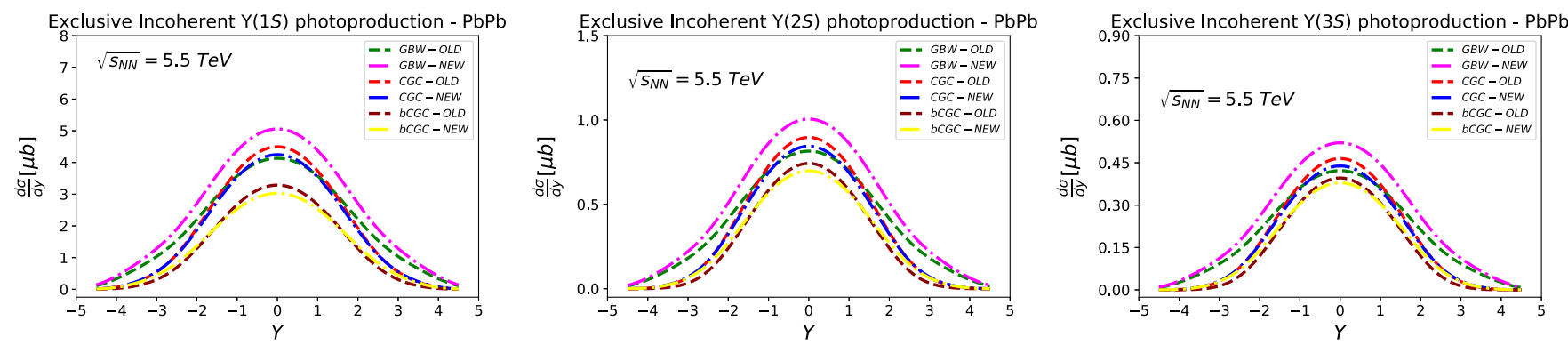

FIG. 3. Exclusive incoherent photoproduction of $\Upsilon(1 S, 2 S, 3 S)$ in $\mathrm{PbPb}$ at $\sqrt{s}=5.5 \mathrm{TeV}$ for the GBW, CGC, and bCGC dipole models. 
calculations, more recent investigations are available in Refs. [23,26]. In Ref. [26] only the coherent $\Upsilon(1 S)$ production has been considered at $5.02 \mathrm{TeV}$. The results are smaller than ours and the main reason is the wave function chosen (the light-cone Gaussian wave function, which gives a smaller overall normalization compared to the boosted Gaussian one). The authors in [26] did not investigate the theoretical uncertainty associated to the wave function or dipole cross sections (only the uncertainty coming from one model for the dipole cross section was addressed). In Ref. [23] the theoretical uncertainty for the coherent and incoherent cross section was investigated. However, predictions for higher energies in $\mathrm{PbPb}$ collisions were not presented and only the $\Upsilon(1 S)$ state was considered (the results are consistent with ours in that case). Finally, we did not consider photonuclear breakup in the present study. We will consider nuclear breackup in a future analysis as it is important and the distinct channels have been measured for $\rho$ and $J / \psi$ photoproduction in UPCs $[27,28]$. This sort of analysis was recently done in Ref. [29], where the coherent $\Upsilon(1 S)$ production was considered using a pQCD model with Next to Leading Order accuracy. An important point discussed in [29] is that the large $y$ region gives the dominant contribution for the $0 \mathrm{nXn}$ and $\mathrm{XnXn}$ channels and they probe larger photontarget center-of-mass energy than the case without neutron tagging.

\section{SUMMARY}

We presented the predictions of rapidity distribution and integrated cross sections for the $\Upsilon(1 S, 2 S, 3 S)$ states for the LHC run 2 energies. The rapidity intervals used in the total cross section were selected to match with the rapidity coverage of LHCb and ALICE detectors both covering $2 \leq y \leq 4.5$. The main contribution is the computation of the incoherent cross section within the color dipole approach and the Glauber-Gribov treatment of nuclear shadowing. The cross sections for the excited states were also calculated in a consistent formalism where the wave function of $2 S$ and $3 S$ states are theoretically well constrained. The usual procedure in the literature involves only an extrapolation of DESY-HERA production ratios to the LHC energies. Our calculations are directly comparable to the STARlight calculation, however distinct procedures are involved in the computation of nuclear shadowing (VDM plus Glauber model versus color dipole plus Glauber-Gribov approach) and how the incoherent cross section is obtained.

\section{ACKNOWLEDGMENTS}

This work was partially financed by the Brazilian funding agency Conselho Nacional de Desenvolvimento Cientifico e Tecnologico (CNPq) and Rio Grande do Sul funding agency Fundação de Amparo À Pesquisa do Estado do Rio Grande do Sul (FAPERGS).
[1] G. Baur, K. Hencken, D. Trautmann, S. Sadovsky, and Y. Kharlov, Phys. Rep. 364, 359 (2002); C. A. Bertulani, S. R. Klein, and J. Nystrand, Annu. Rev. Nucl. Part. Sci. 55, 271 (2005).

[2] N. N. Nikolaev and B. G. Zakharov, Phys. Lett. B 332, 184 (1994); Z. Phys. C 64, 631 (1994).

[3] J. Nemchik, N. N. Nikolaev, E. Predazzi, and B. G. Zakharov, Phys. Lett. B 374, 199 (1996).

[4] F. Gelis, E. Iancu, J. Jalilian-Marian, and R. Venugopalan, Annu. Rev. Nucl. Part. Sci. 60, 463 (2010); H. Weigert, Prog. Part. Nucl. Phys. 55, 461 (2005); J. Jalilian-Marian and Y. V. Kovchegov, Prog. Part. Nucl. Phys. 56, 104 (2006); A. L. Ayala, M. B. G. Ducati, and E. M. Levin, Nucl. Phys. B493, 305 (1997).

[5] A. Dainese, in Proceedings of the 38th International Symposium of Multiparticle Dynamics (ISMD2008): Hamburg, Germany, 2008 (Verlag Deutsches Elektronen-Synchrotron, Hamburg, Germany, 2009), ISBN: 9783935702317 [arXiv:0902.0377].

[6] M. B. G. Ducati, F. Köpp, M. V. T. Machado, and S. Martins, Phys. Rev. D 94, 094023 (2016).

[7] R. Aaij et al. (LHCb Collaboration), J. High Energy Phys. 09 (2015) 084.
[8] K. Dusling, F. Gelis, T. Lappi, and R. Venugopalan, Nucl. Phys. A836, 159 (2010).

[9] T. Lappi, H. Mäntysaari, and R. Venugopalan, Phys. Rev. Lett. 114, 082301 (2015); H. Mäntysaari and B. Schenke, Phys. Rev. Lett. 117, 052301 (2016).

[10] L. Frankfurt, G. A. Miller, and M. Strikman, Phys. Rev. Lett. 71, 2859 (1993); A. Caldwell and H. Kowalski, Phys. Rev. C 81, 025203 (2010).

[11] K. Tuchin, Phys. Rev. C 79, 055206 (2009); T. Lappi and H. Mäntysaari, Phys. Rev. C 83, 065202 (2011); T. Toll and T. Ullrich, Phys. Rev. C 87, 024913 (2013); H. Mäntysaari, B. Schenke, C. Shen, and P. Tribedy, Phys. Lett. B 772, 681 (2017).

[12] J. Cepila, J. G. Contreras, and J. D. T. Takaki, Phys. Lett. B 766, 186 (2017).

[13] S. R. Klein, J. Nystrand, J. Seger, Y. Gorbunov, and J. Butterworth, Comput. Phys. Commun. 212, 258 (2017).

[14] S. Klein and J. Nystrand, Phys. Rev. C 60, 014903 (1999).

[15] B. Z. Kopeliovich and B. G. Zakharov, Phys. Rev. D 44, 3466 (1991); Yu. P. Ivanov, J. Huefner, B. Z. Kopeliovich, and A. V. Tarasov, AIP Conf. Proc. 660, 283 (2003).

[16] J. Nemchik, N. N. Nikolaev, E. Predazzi, and B. G. Zakharov, Z. Phys. C 75, 71 (1997). 
[17] B. E. Cox, J. R. Forshaw, and R. Sandapen, J. High Energy Phys. 06 (2009) 034.

[18] K. Golec-Biernat and M. Wüsthoff, Phys. Rev. D 59, 014017 (1998).

[19] E. Iancu, K. Itakura, and S. Munier, Phys. Lett. B 590, 199 (2004).

[20] H. Kowalski, L. Motyka, and G. Watt, Phys. Rev. D 74, 074016 (2006).

[21] G. Soyez, Phys. Lett. B 655, 32 (2007).

[22] A. H. Rezaeain and I. Schmidt, Phys. Rev. D 88, 074016 (2013).

[23] G. Sampaio dos Santos and M. V. T. Machado, J. Phys. G 42, 105001 (2015).
[24] N. Armesto and A. H. Rezaeian, Phys. Rev. D 90, 054003 (2014).

[25] A. Adeluyi and A. Nguyen, Phys. Rev. C 87, 027901 (2013).

[26] V. P. Gonçalves, B. D. Moreira, and F. S. Navarra, Phys. Rev. D 95, 054011 (2017).

[27] J. Adam et al. (ALICE Collaboration), J. High Energy Phys. 09 (2015) 095.

[28] V. Khachatryan et al. (CMS Collaboration), Phys. Lett. B 772, 489 (2017).

[29] V. Guzey, E. Kryshen, and M. Zhalov, Phys. Rev. C 93, 055206 (2016). 